\title{
Retraction
}

\section{Retracted: A Numerical Model for Torsion Analysis of Composite Ship Hulls}

\author{
Mathematical Problems in Engineering \\ Received 23 October 2013; Accepted 23 October 2013; Published 14 January 2014 \\ Copyright (C) 2014 Mathematical Problems in Engineering. This is an open access article distributed under the Creative Commons \\ Attribution License, which permits unrestricted use, distribution, and reproduction in any medium, provided the original work is \\ properly cited.
}

The paper titled "A Numerical Model for Torsion Analysis of Composite Ship Hulls" [1], published in Mathematical Problems in Engineering, has been retracted as it is found to contain a substantial amount of materials from the previously published papers: (1) Ionel Chirica, Sorin Dumitru Musat, Raluca Chirica, and Elena-Felicia Beznea, "Torsional behaviour of the ship hull composite model," Comput. Mater. Sci., Vol. 50, pp. 1381-1386, 2011; (2) Ion Raluca, Musat Sorin Dumitru, Chirica Ionel, Boazu Doina, and Beznea Elena Felicia, "Torsion analysis of ship hull made of composite materials," Materiale Plastice, Vol. 47, Issue 3, pp. 364-369, 2010; (3) Ion Raluca, Musat Sorin Dumitru, Chirica Ionel, Boazu Doina, and Beznea Elena-Felicia, "Torsional analysis of ship hull model," The Annals of University "Dunarea De Jos" of Galati, Fascicle VIII, 2009 (XV), ISSN 1221-4590, Issue 2, Tribology.

\section{References}

[1] I. Chirica and E.-F. Beznea, "A numerical model for torsion analysis of composite ship hulls," Mathematical Problems in Engineering, vol. 2012, Article ID 212346, 17 pages, 2012. 


\title{
A Numerical Model for Torsion Analysis of Composite Ship Hulls
}

\author{
Ionel Chirica and Elena-Felicia Beznea \\ "Dunarea de Jos" University of Galati, Domneasca Street, 47, 800008 Galati, Romania \\ Correspondence should be addressed to Ionel Chirica, ionel.chirica@ugal.ro \\ Received 17 July 2011; Revised 7 November 2011; Accepted 10 November 2011 \\ Academic Editor: Ming Li \\ Copyright @ 2012 I. Chirica and E.-F. Beznea. This is an open access article distributed under \\ the Creative Commons Attribution License, which permits unrestricted use, distribution, and \\ reproduction in any medium, provided the original work is properly cited. \\ A new methodology based on a macroelement model proposed for torsional behaviour of the \\ ship hull made of composite material is proposed in this paper. A computer program has been \\ developed for the elastic analysis of linear torsion. The results are compared with the FEM-based \\ licensed soft COSMOS/M results and measurements on the scale simplified model of a container \\ ship, made of composite materials.
}

\section{Introduction}

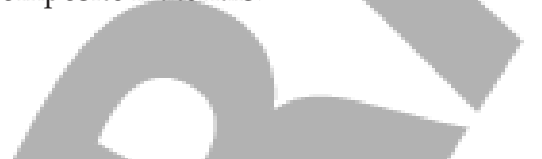

A big interest in improving design, fabrication, and mechanical performance of low-cost composites has led to increase in the use of composites for large patrol boats, hovercrafts, mine hunters, and corvettes. Currently, there are all-composite naval ships up to $80-90 \mathrm{~m}$ long, and this trend continues. It is predicted that hulls for midsized warships, such as frigates that are typically 120-160 m long, may be constructed in composite materials from 2020.

Investigation into the elastic behavior of thin-walled beams with open and closed cross-sections has been carried out since the early works $[1,2]$.

The proliferation of the specialized literature, mainly in the form of journal/proceedings papers [3-7], and the activity in terms of workshops devoted to this topic attest this interest. A decisive factor that has fueled this growing activity was generated by high diversity and severity of demands and operating conditions imposed on structural elements involved in the advanced technology. In order to be able to survive and fulfill their mission in the extreme environmental conditions in which they operate, new materials and new structural paradigms are required.

Monographs written by Kollbrunner and Basler [3] are useful references for developing new methods of the thin-walled beam theory and its applications. The intensive research 
works have been made over the years to develop finite element models that can accurately represent the complex flexural-torsional warping deformable response of these structures. In [4] Yonghao et al. present the theory and method of analyzing horizontal-torsion-coupled dynamic behavior of a ship hull with large hatch opening by the transfer matrix method, considering the ship hull as a simplified free-free nonuniform thin-walled beam.

The estimation of the torsion strength of the ship hull is very important for its structural safety against applied loads [5-9]. Various methodologies have been developed to evaluate the torsion hull girder capacity, [5-9]. The torsion strength obtained by various methods is compared with experimental results and it appears that the proposed methodology is simple yet robust in estimating hull girder torsion strength [10, 11].

Different analytical tools have so far been developed by researches to successfully predict the torsion behaviour associated with ship hull subjected to different loading conditions.

The use of finite-elements analysis for investigation of torsion problem of ship hull is becoming popular due to the improvement in computational hardware and emergence of highly specialized software.

There has been a growing interest in the foundation of the theory of thin-walled composite beams and of their incorporation in civil and naval constructions in the last two decades $[8,9,12]$.

To ensure safe design of a ship's hull, traditionally, the longitudinal strength of the ship hull with length exceeding $60 \mathrm{~m}$ must be assessed during the design stage [13].

The longitudinal failure of ship hulls made of composite materials is usually easier due to the relative low stiffness and relative thin structures. With the trend that the size of ship hull in composite materials is upon large scale, it is becoming necessary to study the longitudinal strength of ship hull made of this type of advanced materials.

Ship hull structure can be considered as thin-walled structures. Plates and shells have one physical dimension, their thickness, small in comparison with their other two. In thin/thick-walled beams all three dimensions are of different order of magnitude. For such structures the wall thickness is small compared with any other characteristic dimension of the cross-section, whereas the linear dimensions of the cross-section are small, compared with the longitudinal dimension.

Ship hulls in composite materials can usually be regarded as assemblies of a series of thin-walled stiffened composite panels. Thus, knowing the strength of stiffened composite panels it is possible to estimate the longitudinal strength of ship hulls in composite materials.

In the paper, the model of a macroelement used for torsion analysis is presented. The macroelement is concerning longitudinal strips plates. The strip plate is treated as an EulerBernoulli plate, because the stretching-compression, bending, and shearing occur due to the torsion of macroelement.

One of the first approach close to the longitudinal strip plates is the folded plate. The theory of folded plate is presented in $[14,15]$. The folded plate method is normally limited to assemblages of rectangular plates. Folded plates are assemblies of flat plates rigidly connected together along their edges in such a way so as to make the structural system capable of carrying loads without the need for additional supporting beams along mutual edges. Each plate is assumed to act as a beam in its own plane.

The strip plate is treated with the same theory as the folded plate but the theory is developed in the context of the thin-walled beam theory for torsion analysis.

According to the thin-walled beam theory, the loading is not acting transversal to the strips, which is the case of folding plate. 


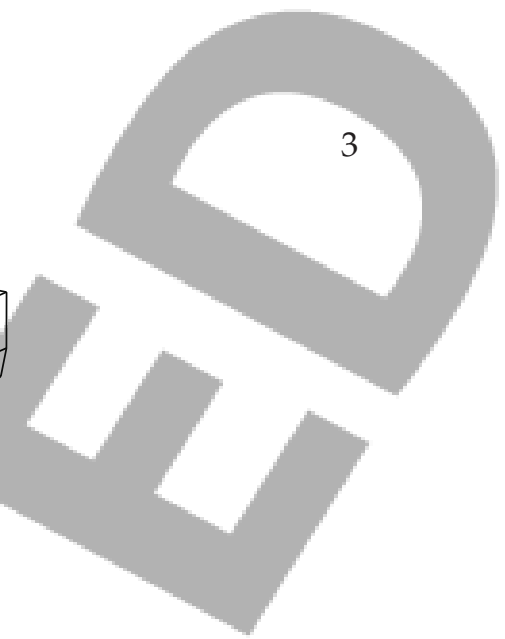

Figure 1: Macroelement model.

Due to their wide applications in civil, aeronautical/aerospace, and naval engineering, and due to the increased use in their construction of advanced composite material systems, a comprehensive theory of thin-/thick-walled beams has to be developed: this is one of the aims of this paper.

The aim of the work is to analyze the influence of the very large open decks on the torsion behaviour of the ship hull made of composite materials.

\section{Ship Hull Macroelement Model}

The ship hull is modelled with macroelements, [16-19] having the transversal section as a thin-walled structure. The outline of the macroelement section is considered as a polygonal one. In the theory, the material is considered as an orthotropic one. For a straight line portion of cross-section outline is corresponding a longitudinal strip plate (Figure 1). Due to the torsion of the thin-walled beam, in the strip plate the stretching-compression, bending, and shearing occur. The strip plate is treated as an Euler-Bernoulli plate. The stiffness matrix of the macro-element is obtained by assembling the stiffness matrices of the strips.

Two coordinate systems are used:

(i) global system $O_{o} X Y Z$ having the axis $O_{o} X$ along the torsion centers line of the cross-sections of the beam.

(ii) Local system attached to each strip plate $k\left(F_{k}^{0} x_{k} y_{k} z_{k}\right)$ having the axis $F_{k}^{0} x_{k}$ parallel with the global axis $O_{0} X$.

The torsion behaviour of the thin-walled beam is depending on the section type. So, the methodology presented in this paper is treating in different ways and different hypothesis, depending on the type of cross section: open and closed.

The open cross section is treated based on the hypothesis of the Vlasov theory [2].

(i) The material is linear-elastic, homogeneous, isotropic generally, having the coordinate system $F_{k}^{0} x_{k} y_{k} z_{k}$ as the main orthotropic axis.

(ii) The shear stresses occurring in the beam cross section are parallel with the median line $\Gamma$.

(iii) During the deformation the median line $\Gamma$ does not remain plane. The projection of the median line on the cross section plane remains the same as its initial shape 


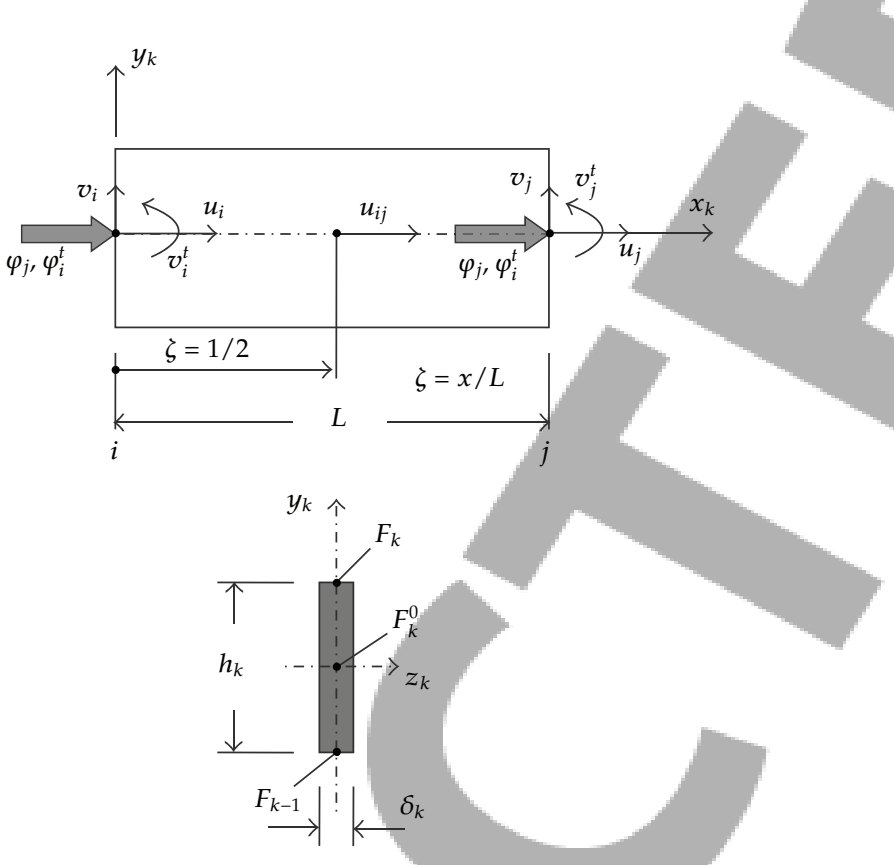

Figure 2: Element of finite strip.

(nondeformed outline hypothesis). For small displacements, the displacement $v$ of the current point $F$ placed on the median line has the equation

$$
v_{k}(x, s)=r_{k}(s) \varphi(x)
$$

(iv) The displacement $u$ along the axis $O_{o} X$ of the point $F$ is considered as constant on the wall thickness. The displacement $u$ is considered to be in the form

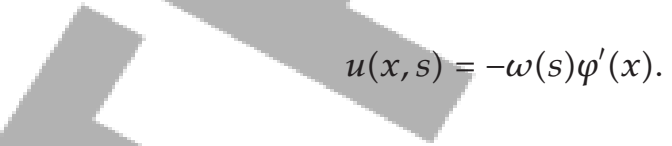

The sectorial coordinate is defined as

$$
\omega(s)=\int_{\Gamma} \tilde{r}(s) \mathrm{d} s
$$

The torsion of the thin-walled beam generates the torsion of the strips and the loading of the strips in their plane (Figure 2).

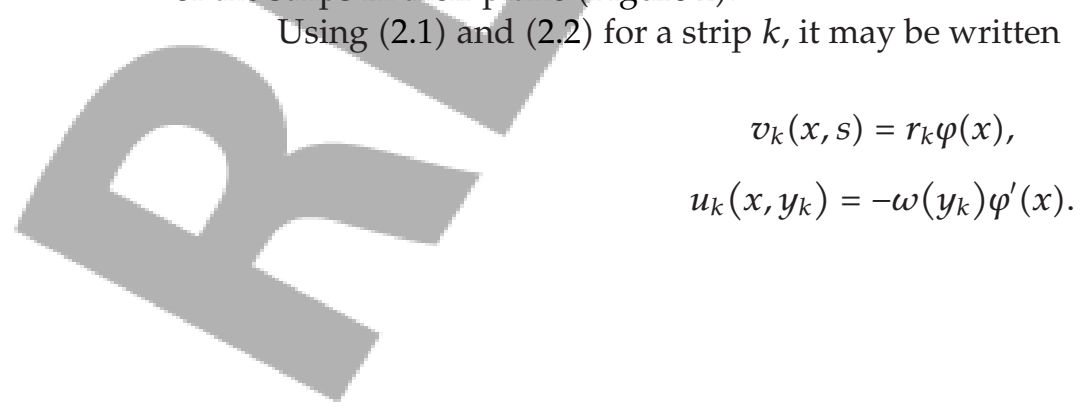




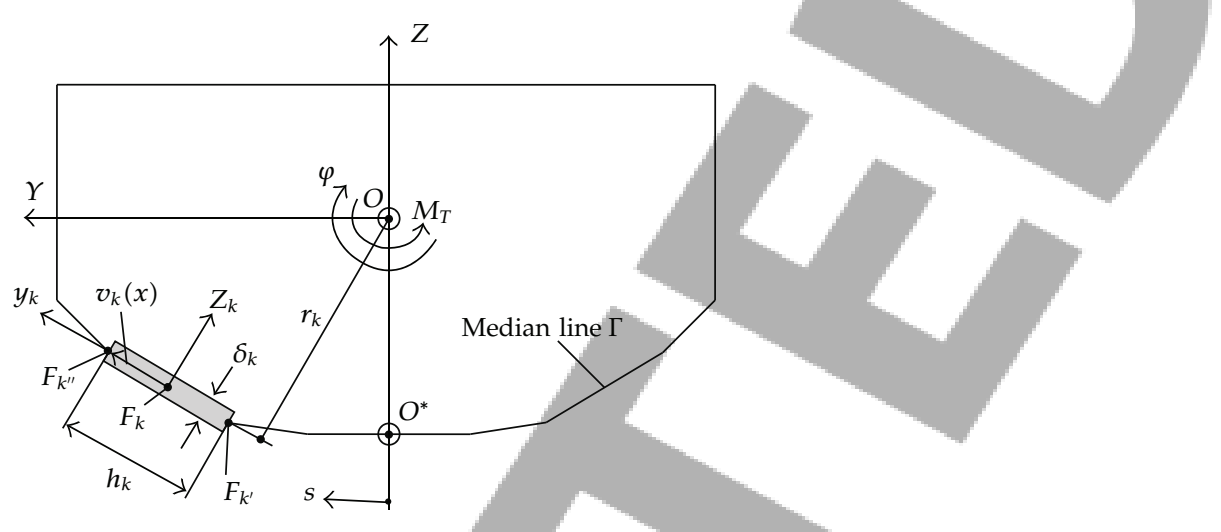

Figure 3: Closed cross section of the macroelement.

For the displacement $u$, due to the tension-compression loading of the strip $k$ the approach function is a parabolic one, having the form

$$
u_{k}(\xi)=P_{1}(\xi) u_{i}^{k}+P_{2}(\xi) u_{i j}^{k}+P_{3}(\xi) u_{j}^{k}
$$

Equation (2.5) is used for the strip-plate matrices determination and also to estimate the maximum stresses in the strip volume, after the macroelement d.o.f. are solved.

For the case of closed section, is considered that $u$ is proportional to the generalized sectorial coordinate $\widehat{\omega}$ evaluated related to $O$ and $O^{*}$. In Figure 3 the parameters to determine the characteristics of the macroelement are presented.

Also, different from classical theory or Benscoter theory, it is consider that $u$ is proportional to the rate of twist

$$
u(x, s)=-\widehat{\omega}(s) \varphi^{\prime}(x) .
$$

The generalized sectorial co-ordinate is defined as

$$
\widehat{\omega}=\omega-\tilde{\omega}
$$

where

$$
\omega(s)=\int_{0}^{s} r(s) \mathrm{d} s ; \quad \omega_{0}=\int_{\Gamma} r(s) \mathrm{d} s ; \quad \tilde{s}=\int_{0}^{s} \frac{\mathrm{d} s}{\delta(s)} ; \quad \tilde{\omega}=\frac{\omega_{0} \tilde{s}}{\tilde{S}} .
$$

(v) The double of the area surrounded by $\Gamma$ is

$$
\widetilde{S}=\int_{\Gamma} \frac{\mathrm{d} s}{\delta(s)}=\sum_{k=1}^{n} \frac{h_{k}}{\delta_{k}},
$$

where $n$ is the number of strip-plates. 
The torsion loading of the beam generates an in-plane loading of the strip-plate. For each strip-plate, it may be written

$$
\begin{gathered}
v_{k}(x)=r_{k} \varphi(x), \\
u_{k}\left(x, y_{k}\right)=-\widehat{\omega}\left(y_{k}\right) \varphi^{\prime}(x) .
\end{gathered}
$$

These equations define the displacement field for each strip-plate. The continuity of the displacement $u$ along the jointing edges between two strip-plates is embedded in the above relation. The linear variation of $\widehat{\omega}_{k}$, the generalized sectorial coordinate along the axis $y_{k}$ (in the reference system $F_{k} x_{k} y_{k} z_{k}$ associated to strip-plate $k$ ) may be expressed as

$$
\widehat{\omega}=\widehat{\omega}_{k}+\left(\widehat{\omega}_{k^{\prime \prime}}-\widehat{\omega}_{k^{\prime}}\right) \eta
$$

where $-1 / 2 \leq \eta=y_{k} / h_{k}<1 / 2$.

The coordinates $\widehat{\omega}_{k}, \widehat{\omega}_{k^{\prime}}$, and $\widehat{\omega}_{k^{\prime \prime}}$ characterize the points, $F_{k}, F_{k^{\prime}}$, and $F_{k^{\prime \prime}}$. The dependent equation between these coordinates is

$$
\widehat{\omega}_{k}=\frac{\widehat{\omega}_{k^{\prime}}+\widehat{\omega}_{k^{\prime \prime}}}{2}
$$

The differential equation of the twist angle $\varphi$ obtained by the Ritz method is

$$
E I_{\widehat{\omega}} \varphi^{\prime \prime \prime}-G I_{T} \varphi^{\prime}=-M_{T}(x),
$$

where

$$
I_{\widehat{\omega}}=\sum_{k=1}^{n} I_{\hat{\omega} k} ; \quad I_{\widehat{\omega} k}=h_{k} \delta_{k}\left[\widehat{\omega}_{k}^{2}+\frac{\left(\widehat{\omega}_{k^{\prime}}-\widehat{\omega}_{k^{\prime \prime}}\right)^{2}}{12}\right]
$$

is sectorial moment of inertia and

$$
I_{T}=\frac{\omega_{0}^{2}}{\widetilde{S}}
$$

is conventional polar moment of inertia; $M_{T}$ is the transmitted torque.

For the displacements $\varphi(\xi)$ and $v_{k}(\xi)$ third-order polynomial functions are chosen:

$$
\begin{gathered}
\varphi(\xi)=H_{1}(\xi) \varphi_{i}+L H_{3}(\xi) \varphi_{i}^{\prime}+H_{2}(\xi) \varphi_{j}+L H_{4}(\xi) \varphi_{i}^{\prime} \\
v_{k}(\xi)=H_{1}(\xi) v_{i}^{k}+L H_{3}(\xi) \theta_{i}^{k}+H_{2}(\xi) v_{j}^{k}+L H_{4}(\xi) \theta_{j}^{k}
\end{gathered}
$$

The polynomial functions are used for the strip-plate matrices determination and also to estimate the maximum stresses in the strip-plate volume, after the macroelement d.o.f. are solved. 
For bending and torsion, the well-known matrices of the beam are obtained,

$$
\mathbf{k}_{v}^{k}=\frac{E I_{k}}{L^{3}}\left[\begin{array}{rrrr}
12 & 6 L & -12 & 6 L \\
& 4 L^{2} & -6 L & 2 L^{2} \\
& 12 & -6 L \\
\text { symm. } & & 4 L^{2}
\end{array}\right] ; \quad \mathbf{k}_{\varphi}^{k}=\frac{G I_{T_{k}}}{L}\left[\begin{array}{rrrr}
\frac{6}{5} & \frac{L}{10} & -\frac{6}{5} & \frac{L}{10} \\
& \frac{2 L^{2}}{15} & -\frac{L}{10} & -\frac{L^{2}}{10} \\
& \frac{6}{5} & -\frac{L}{10} \\
& & \frac{2 L^{2}}{15}
\end{array}\right] .
$$

The strips' stiffness matrix from axial and bending loading are assembling to realize the stiffness matrix of the macroelement. The assembling process was performed by transforming process using the orthogonal process, presented in [20]:

$$
\mathbf{K}=\frac{E I_{\omega}}{L^{3}} \tilde{\mathbf{K}}_{\mathrm{EB}}+\frac{G I_{T}}{L} \tilde{\mathbf{K}}_{\mathrm{SV}}
$$

where

$$
\tilde{\mathbf{K}}_{\mathrm{EB}}=\left(\begin{array}{cccc}
12 & 6 L & -12 & 6 L \\
& 4 L^{2} & -6 L & 2 L^{2} \\
& \text { symm. } & 12 & -6 L \\
& & & 4 L^{2}
\end{array}\right),
$$

$$
\tilde{\mathbf{K}}_{\mathrm{SV}}=\left(\begin{array}{cccc}
\frac{6}{5} & \frac{L}{10} & -\frac{6}{5} & \frac{L}{10} \\
\frac{2 L^{2}}{15} & -\frac{L}{10} & -\frac{L^{2}}{30} \\
& & \frac{6}{5} & -\frac{L}{10} \\
& & \frac{2 L^{2}}{15}
\end{array}\right) .
$$

Finally, the equation of macroelement is

$$
\mathbf{K} \Delta+\mathbf{F}=\mathbf{0},
$$

where $\Delta$ is vector of displacements and $\mathbf{F}$ is vector of external load (torques).

\section{Equivalent Stiffness Characteristics of the Composite Shell}

In the methodology, the classical thin-walled beam theory for isotropic materials was used. Due to the fact the material used in the fabrication of the ship hull, the orthotropy of the material is to consider. 


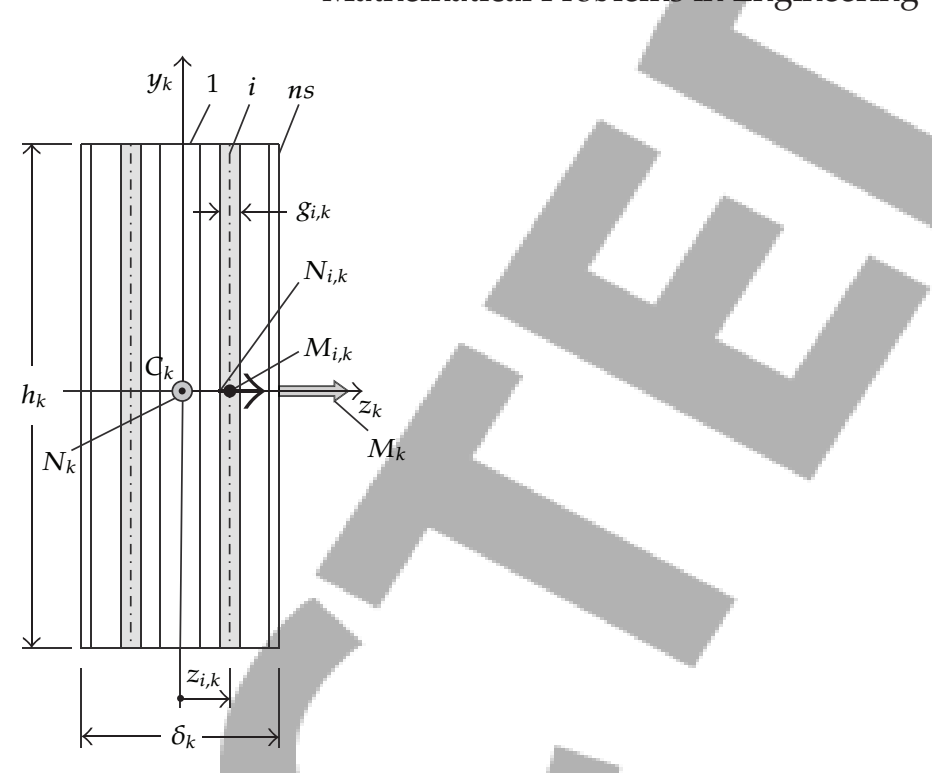

Figure 4: Strip thickness section. Stretching and bending of the strip.

The orthotropy system of the strips is considered to be $F_{k}^{0} x_{k} y_{k} z_{k}$. Also, it is considered that the stack of layers may be different from one to other layer. The stack is considered as a symmetric one, $2 \mathrm{~ns}$ being the number of layers.

The equivalent stiffness coefficients for the tension-compression, bending, and shearing loading of the strip $k$ are determined according to the equation of static equilibrium.

The points placed in a layer are considered in a plane state in the plane $F_{k}^{0} x_{k} y_{k}$.

The hypothesis of undeformed cross-section $\left(\sigma_{y}=0\right)$, leads to the following equation $[6,7]$ for the strip $k$ and layer $i$

So

$$
\left(\begin{array}{c}
\sigma_{x} \\
\tau_{x y}
\end{array}\right)_{i, k}=\left(\begin{array}{cc}
\bar{Q}_{11} & 0 \\
0 & \bar{Q}_{66}
\end{array}\right)_{i, k}\left(\begin{array}{c}
\varepsilon_{x} \\
\gamma_{x y}
\end{array}\right)_{i, k} .
$$

For the tension-compression of a strip $k$ (Figure 4) (having the longitudinal displacement $u_{k}$ ) the following equations may be written as follows.

(i) For each layer $i$

$$
A_{i, k}=g_{i, k} h_{k}, \quad N_{i, k}=E_{i, k} A_{i, k} u_{k}^{\prime} .
$$

(ii) For the strip considered as homogeneous one,

$$
N_{k}=(E A)_{k} u_{k}^{\prime} .
$$


Mathematical Problems in Engineering

(iii) From the condition of equivalence of the axial force

$$
N_{k}=2 \sum_{i=1}^{\mathrm{ns}} N_{i, k}
$$

The equivalent longitudinal stiffness is obtained

$$
(E A)_{k}=2 \sum_{i=1}^{\mathrm{ns}}\left(E_{i, k} g_{i, k}\right) h_{k}
$$

For the bending of a strip $k$ (Figure 4) (having the transversal displacement $v_{k}$ ) the following equations may be written as follows.

(i) For each layer $i$

$$
I_{i, k}=\frac{g_{i, k} h_{k}^{3}}{12}, \quad M_{i, k}=-E_{i, k} I_{i, k} v_{k}^{\prime \prime} .
$$

(ii) For the strip considered as homogeneous one,

$$
M_{k}=-(E I)_{k} v_{k}^{\prime \prime}
$$

(iii) From the condition of equivalence of the bending moment

$$
M_{k}=2 \sum_{i=1}^{\mathrm{ns}} M_{i, k}
$$

the equivalent bending stiffness is obtained

$$
(E I)_{k}=2 \sum_{i=1}^{\mathrm{ns}} E_{i, k} I_{i, k}
$$
follows.

For the shearing of the strip $k$ (Figure 5) the following equations may be written as

(i) For each layer $i$

$$
\tau_{i, k}=G_{i, k} \gamma_{k}, \quad T_{i, k}=G_{i, k} g_{i, k} h_{k} \gamma_{k} .
$$

(ii) For the strip considered as homogeneous one,

$$
T_{k}=(G A)_{k} \gamma_{k}
$$




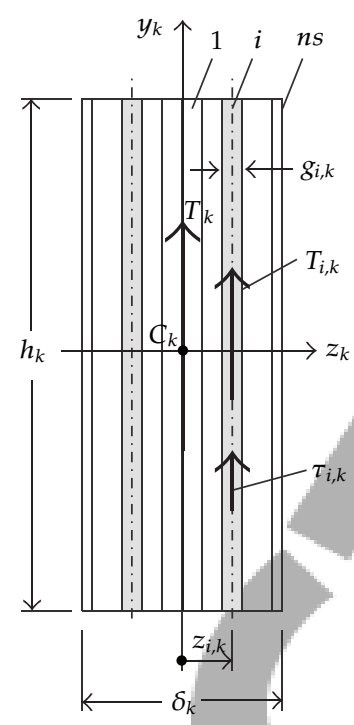

Figure 5: Strip thickness section. Determination of equivalent shear stiffness.

(iii) From the condition of equivalence of the shear force

$$
T_{k}=2 \sum_{i=1}^{\mathrm{ns}} T_{i, k}
$$

the equivalent shearing stiffness is obtained

$$
(G A)_{k}=2 \sum_{i=1}^{\mathrm{ns}} G_{i, k} g_{i, k} h_{k}
$$

By introducing the hypothesis of the same geometry of the equivalent section (same $h_{k}$ and $\delta_{k}$ ) from (3.10) is resulting the equivalent Young's modulus for strip

$$
E_{k}=\frac{2}{\delta_{k}} \sum_{i=1}^{\mathrm{ns}} E_{i, k} g_{i, k}
$$

and from (3.14) the equivalent shear modulus for strip $k$,

$$
G_{k}=\frac{2}{\delta_{k}} \sum_{i=1}^{\mathrm{ns}} G_{i, k} g_{i, k}
$$




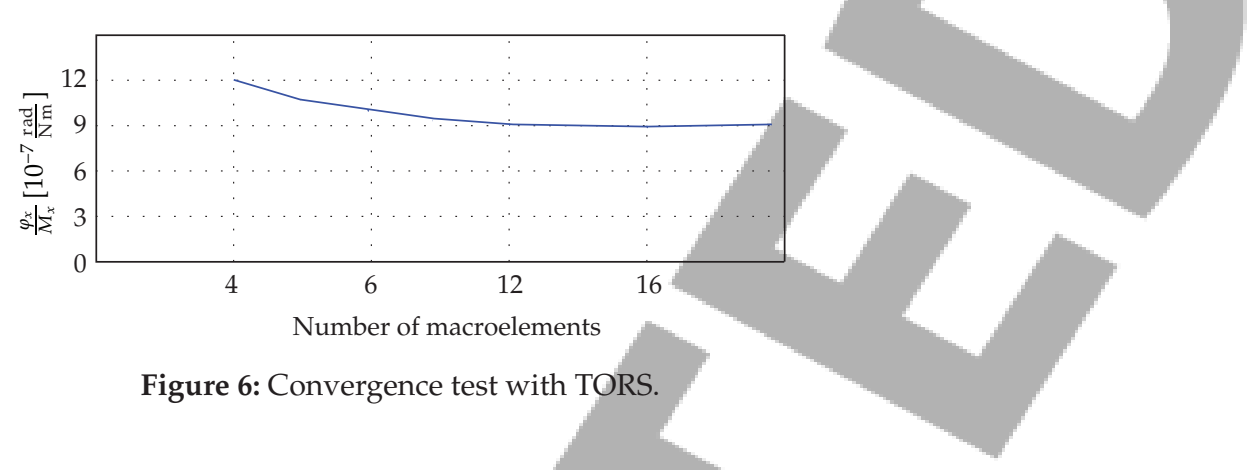

\section{Numerical Analysis of the Ship Hull Model}

\subsection{Numerical Modeling with Macroelements, Using the New Code TORS}

Based on the numerical methodology, presented in previous section, a new code TORS, written in MATHLAB, was developed for torsion analysis of ship hull.

Taking into account the proposed methodology is based on the thin-walled beam hypothesis, only longitudinal elements are considered in torsion calculus. In this section, the proposed methodology is applied on a simplified model of a container ship, having especially longitudinal shells, connected in between by transversal bulkheads.

The simplified container ship hull model has the main characteristics: length $L=2.4 \mathrm{~m}$, breadth $B=0.4 \mathrm{~m}$, and depth $D=0.2 \mathrm{~m}$.

The material is E-glass/polyester having the symmetric stack. The stack of the shell is according to the topologic code $[\mathrm{A} / \mathrm{B}] 3 \mathrm{~s}$. The stack of the deck and bulkheads is according to the topologic code $[\mathrm{A} / \mathrm{B} / \mathrm{A} / \mathrm{B} / \mathrm{A} / \mathrm{B} / \mathrm{A} / \mathrm{B} / \mathrm{A}] \mathrm{s}$.

The layers made of material $A$, have the thickness of $0.25 \mathrm{~mm}$ and the following characteristics:

$$
E_{x}=80 \mathrm{GPa}, \quad E_{y}=80 \mathrm{GPa}, \quad G_{x y}=10 \mathrm{GPa}, \quad \mu_{x y}=0.2 .
$$

The layers made of material $\mathrm{B}$, have the thickness of $0.1 \mathrm{~mm}$ and the following characteristics:

$$
E_{x}=3.4 \mathrm{GPa}, \quad E_{y}=3.4 \mathrm{GPa}, \quad G_{x y}=1.3 \mathrm{GPa}, \quad \mu_{x y}=0.3 .
$$

According to the equations from Section 3, the equivalent mechanical characteristics of the composite are

$$
E_{x}=58.11 \mathrm{GPa} ; \quad E_{y}=58.11 \mathrm{GPa} ; \quad G_{y x}=7.51 \mathrm{GPa} ; \quad \mu_{x y}=0.228 .
$$

To fulfill the hypothesis of no deformability of the cross section, transversal bulkheads are placed at every $200 \mathrm{~mm}$. The middle part of the model has a narrow deck, as it is exists on the real ship to easy charging.

To determine the number of macroelements for mesh, a convergence study was done. So, for each element the same longitudinal dimension was chosen. As it is seen, in Figure 6, the minimum number of macroelements since the maximum value of the torsion angle remains the same, 12 . 


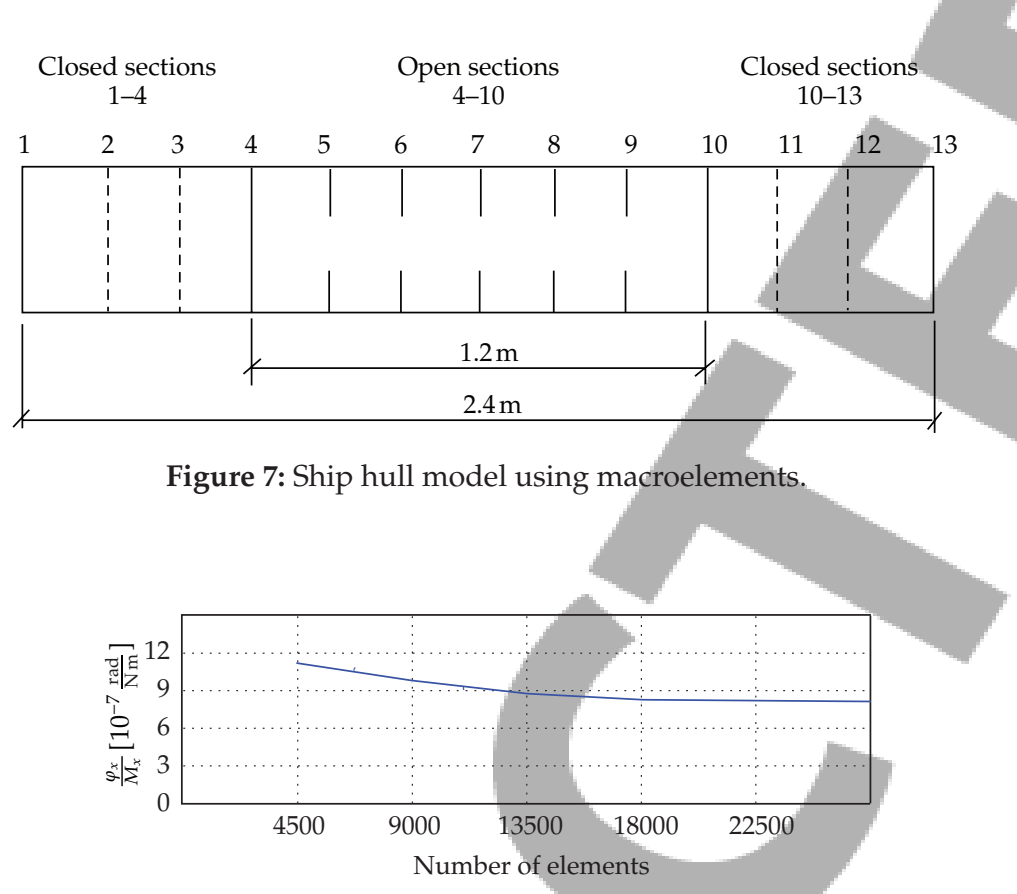

Figure 8: Convergence test with COSMOS/M.

For the torsion analysis the numerical model using TORS the mesh concerns 12 macroelements (6 macroelements in the open area of the ship hull and 6 macroelements in the closed part of the ship hull as it is presented in Figure 7). Each macroelement represents a part of the ship hull placed between two transversal bulkheads. For one macroelement, longitudinal strip plates to describe the ship hull part were used. In the open area 12 strip plates are used (according to Figure 1). For the macroelements with closed form 16 longitudinal strip plates are used. For all macroelements, the bilge is modeled with 3 longitudinal strip plates. The model was considered as clamped on the end sections and the loading was developed by a unitary torque applied in the midship section (section number 7, in Figure 7).

The results of the numerical analysis obtained with TORS are presented in Section 5, where a comparative discussion on the results is presented.

\subsection{Numerical Modeling with FEM, Using Software COSMOS/M}

The compared second numerical torsion of the simplified container ship hull model was done using SHELL3L finite plate elements from the licenced package software COSMOS/M.

To determine the number of elements for FEM mesh, a convergence study was done.

As it is seen, in Figure 8, the minimum number of elements since the maximum value of the torsion angle remains the same, is 18110 . The side dimension of a triangle plate element is $0.02 \mathrm{~mm}$. In Figure 9, the deformed shape of the model is presented.

The model was considered as clamped on the end sections and the loading was developed by a unitary torque applied on the midship section. 


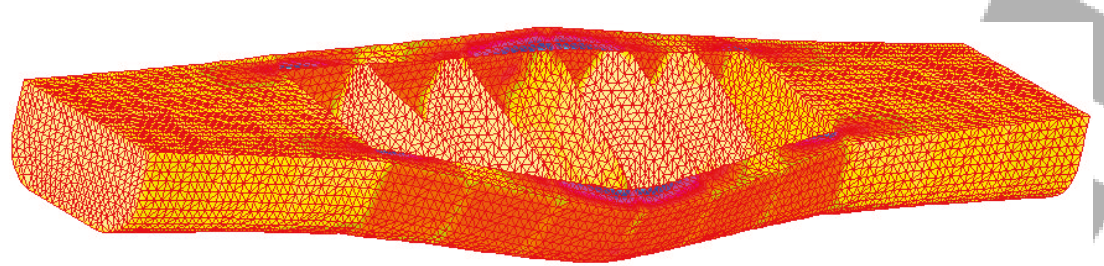

Figure 9: Torsion deformation of the ship hull model.

Table 1: The CPU time of solving module.

Method CPU time

(1) The partial-pivoting Gaussian elimination scheme $30.46 \mathrm{~s}$

(2) Classic LU decomposition $35.89 \mathrm{~s}$

(3) Iterative method Jacobi $55.70 \mathrm{~s}$

(4) Optimized LU decomposition (Lapack) $31.59 \mathrm{~s}$

(5) Leverrier algorithm $\quad 66.32 \mathrm{~s}$

COSMOS/M $88.00 \mathrm{~s}$

The results of the numerical analysis obtained with FEM package software COSMOS/M are presented in Section 5, where a comparative discussion on the results is presented.

\subsection{CPU Time Appreciation}

The resolution of (2.20) (the unknown is the displacement vector $\boldsymbol{\delta}$ ) makes it possible to find the torsion deformation of the ship hull structure.

We have tried five methods to calculate the determinant of the stiffness matrix. Table 1 summarizes the CPU time machine necessary to find the solution of the numerical ship hull model.

According to the benchmarking analysis of solver used for numerical modeling with macroelements, using the new code TORS the partial-pivoting Gaussian elimination scheme was chosen for TORS.

In Table 1 the CPU time machine necessary to find the solution of the numerical ship hull model with FEM in COSMOS/M is bigger than the CPU time obtained in TORS.

\section{Experimental Validation}

The simplified container ship hull model was built by E-glass/polyester having the characteristics presented in Section 4.1.

The experimental analysis of the ship model was done with a system concerning the clamping system and loading rig used to carry out torque tests.

The ship hull model is loaded by a torque $M_{x}$ applied in the midship section. The torque is developed by a special system concerning two frames and two hinged bars (Figure 10). A very rigid frame, having two arms, is placed in the midship section (Figure 11). Each arm is hinged, by an intermediate dynamometer with rigid bar by another rigid frame fixed on the floor. The couple of forces are developed by two thrusts placed on the end of each 

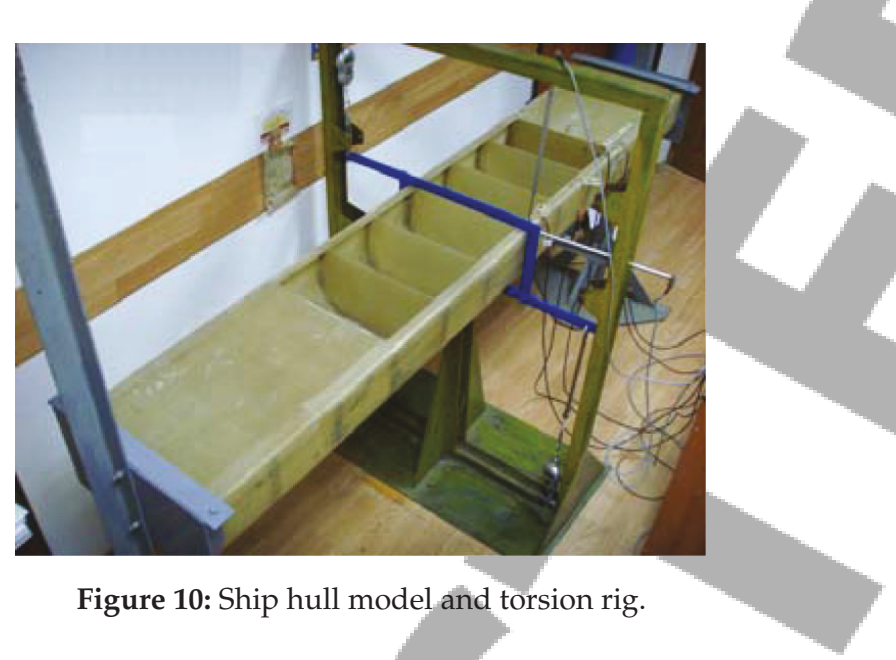

Figure 10: Ship hull model and torsion rig.

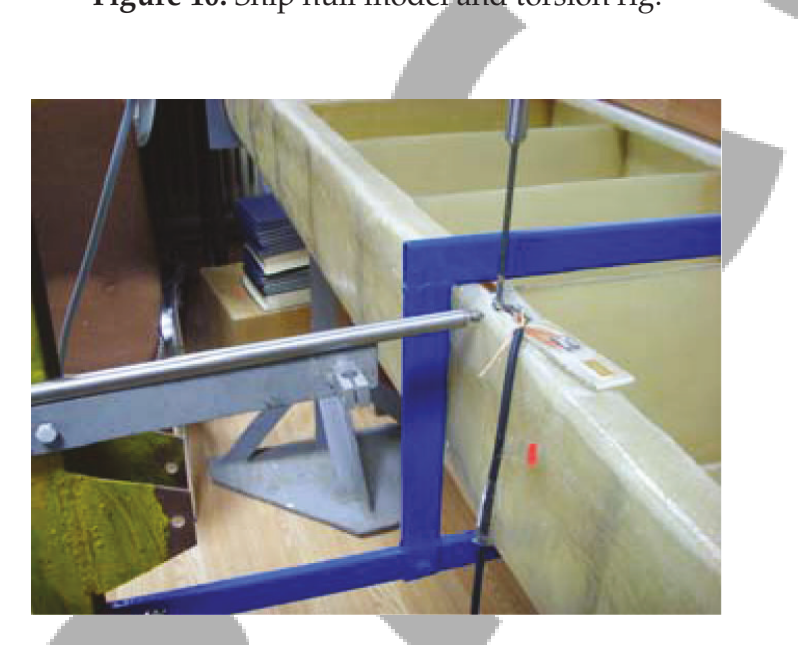

Figure 11: Deformation measurement rig.

bar. Due to the fact that the real ship has much stiffened structure on the both ends, from the point of view of torsion the model is clamped at the ends.

The torsion deformation of the cross section was determined by measuring the vertical and horizontal displacements of the point placed at the intersection between deck and side shell, determined with displacement transducers (LVDT system).

According to the torsion rotations (Figure 12) obtained with FEM calculus, the symmetry regarding midship section is observing. The same characteristic is obtained for the warping stress (Figure 13) in the cross section.

The calculus with TORS is producing the maximum deviation from the torsion rotation symmetry of $1.65 \%$. The maximum deviation for the warping stress symmetry in midship section is of $1.5 \%$.

The variation of the relative torsion angle $\left(\varphi_{x} / M_{x}\right)$ along the ship model obtained so in FEM analysis, macroelement model, and experimental measurements is presented in Figure 12. Due to the closed section type in the ends, the torsion stiffness of the model in these areas is much higher than in the middle part. As it is seen in Figure 12, the maximum value of the relative torsion angle $\left(\varphi_{x} / M_{x}\right)$ in the midship is almost 2-times more than the maximum torsion angle in the closed area. 


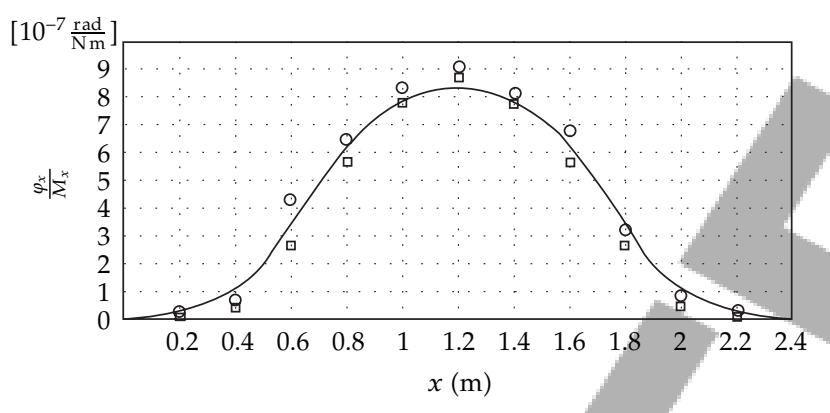

$$
\begin{array}{ll}
\square & \text { TORS } \\
\bigcirc & \text { Experiment } \\
& \text { FEM analysis }
\end{array}
$$

Figure 12: Relative torsion angle variation along the ship hull model.

Figure 13: Relative normal stress (warping stress) in the midship section [Pa/Nm].

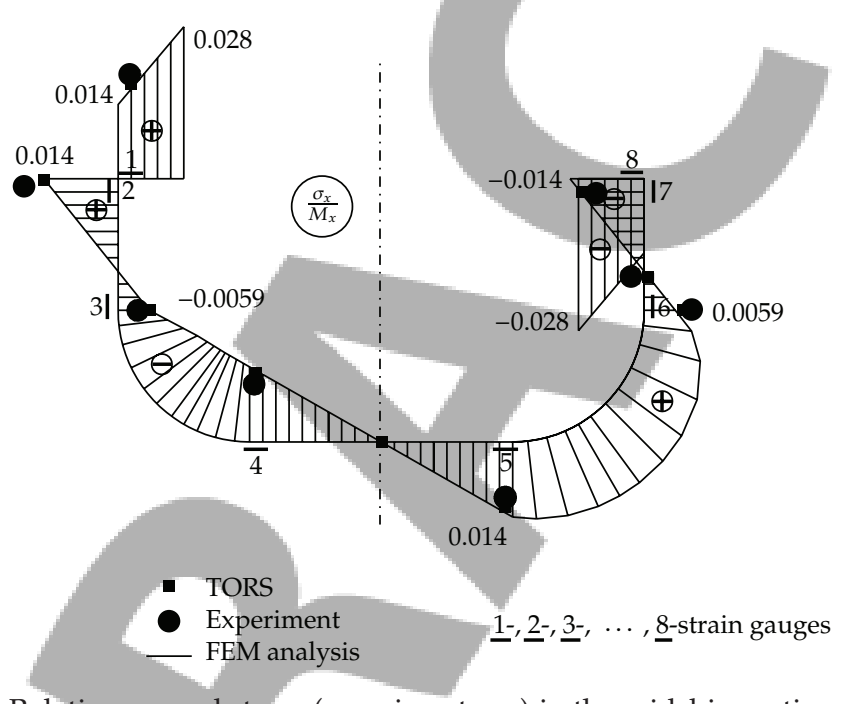

. 13: Relative normal stress (warping stress) in the midship section [ $\mathrm{Pa} / \mathrm{Nm}]$

The numerical results obtained with the code TORS, based on this method, are compared with the results obtained with FEM analysis COSMOS/M and experimental results on the model of a ship with large open deck.

Due to the variation of the cross section shape of the model, a coupled torsion with lateral bending occurred.

In spite of the fact that the torque is applied so that it can provide a pure torsion, warping of the sections occurs. Warping of the section is only depending on the section geometry which means that along the model exist free warping and restrained warping sections.

The values obtained with FE analysis and according to the strain gauges measurements are presented.

The variation of the relative normal stress in the midship open section is presented in Figure 13. In the figure, the results obtained with TORS (macroelement model) FEM analysis and in experimental tests for the 8 points of section are presented. Due to the fact 
Table 2: Maximum deviation (\%) of the TORS results versus FEM analysis and experiments results.

\begin{tabular}{cccc}
\hline \multicolumn{2}{c}{ TORS-FEM } & \multicolumn{2}{c}{ TORS-experiment } \\
$\operatorname{Max}\left|\Delta\left(\sigma_{x} / M_{x}\right)\right|$ & $\operatorname{Max}\left|\Delta \varphi_{x} / M_{x}\right|$ & $\operatorname{Max} \mid \Delta\left(\sigma_{x} / M_{x} \mid\right.$ & $\operatorname{Max}\left|\Delta \varphi_{x} / M_{x}\right|$ \\
\hline 2.51 & 2.76 & 2.66 & 2.95 \\
\hline & &
\end{tabular}

the variation of the normal stress is linear type, the variation of the ratio $\left(\sigma_{x} / M_{x}\right)$ was plotted with continuum line. The values of the stresses obtained with strain gauges are plotted in the figure.

The hypothesis of thin-walled beam is not totally fulfilled by the TORS. This is the reason of deviation of the results obtained with the methodology-based TORS.

The differences between the results obtained in the three methods are presented in Table 2. As it is seen, the maximum deviation of the results obtained with the methodology used in TORS is acceptable and generally is placed under 3\%.

\section{Concluding Remarks}

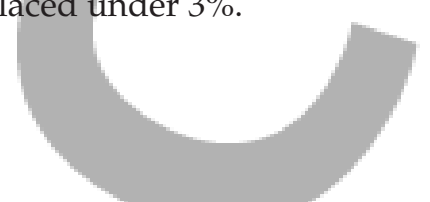

The methodology presented in this paper concerns a proposed macroelement model of thinwalled beam for torsion analysis of ship hull made of composite material. The assumptions concerning the material properties and the stress distribution in the beam have been made.

A finite element computer program TORS has been developed for the elastic analysis of linear torsion. For this program, the ship hull beam is divided into a number of macroelements. Each macroelement is divided into a number of strip elements, each of which has a cubic variation of the twist rotation $\varphi$ along its length. The data for each strip element consists of the characteristics of composite (stack and material).

Each element may have a uniformly distributed torque per unit length $m_{x}$, while concentrated torques $M$ and bimoments $B$ may act at the nodes between macro elements. Each node may have restraints which prevent twist rotation and/or warping.

The hypothesis of thin-walled beam is not totally fulfilled by the methodology applied in TORS and so the deviation of the results obtained with the code TORS can be explained.

As it is seen in Table 2 the maximum deviation of the results obtained with the methodology used in TORS is placed under $3 \%$.

The tests on the thin-walled beam theory fulfillment by the proposed methodology were done for joining between transversal bulkheads (membranes) and side shells. So, these joints were studied by considering pendulums or welding. The model with joints considered as pendulums is less rigid than the model with welded bulkheads. The loading of the transversal bulkheads is not important in the case of torsion.

The results obtained by the three methods (macroelement model-TORS software, FEM-COSMOS/M software, and experimental tests) are in a fair agreement.

The facile mode to prepare the input data for defining the cross section, by using macroelement model and code TORS, recommends the methodology as a good tool for torsion analysis of ship hull made of composite materials. Additionally, the CPU time machine with TORS is much lesser than the CPU time obtained with COSMOS/M. 
Mathematical Problems in Engineering

\section{Acknowledgment}

This paper has been supported by UEFISCDI, project number PNII-IDEI code 512/2008

(2008-2011).

\section{References}

[1] S. P. Timoshenko and J. M. Gere, Theory of Elastic Stability, McGraw-Hill, New York, NY, USA, 2nd edition, 1961.

[2] V. Z. Vlasov, Thin-Walled Elastic Beams, Program of Scientific Translations, Jerusalem, Israel, 2nd edition, 1961.

[3] C. F. Kollbrunner and K. Basler, Torsion in Structures, Springer, Berlin, Germany, 1969.

[4] P. Yonghao, T. Yougang, and Y. Xueyong, "The horizontal-torsion-coupled model analysis and dynamic response calculation of the ship hull with a large hatch openign," Journal of Tianjin University, pp. 51-58, 1993.

[5] P. T. A. Pedersen, "Beam model for the torsional-bending response of ship hull," Transactions of Royal Institution of Naval Architects, pp. 267-275, 1982.

[6] I. Senjanović and Y. Fan, "A higher-order theory of thin-walled girders with application to ship structures," Computers and Structures, vol. 43, no. 1, pp. 31-52, 1992.

[7] I. Senjanović, S. Tomašević, and N. Vladimir, "An advanced theory of thin-walled girders with application to ship vibrations," Marine Structures, vol. 22, no. 3, pp. 387-437, 2009.

[8] I. Senjanovic, N. Vladimir, and T. Marko, "Investigation of torsion, warping and distortion of large container ships," Ocean Systems Engineering, vol. 1, no. 1, pp. 73-93, 2011.

[9] N. Wu and M. Chun-yan, "A comparative study on the hull strength of a ship with thin-walled box gird-ers," Journal of Marine Science and Application, vol. 5, no. 3, pp. 11-16, 2006.

[10] T. Kawai and Y. Fujitani, "Some considerations on the modern beam theory-development of the practical methods," Report of the Institute of Industrial Science, University of Tokyo, vol. 32, no. 4, 1986.

[11] Y. Hu, X. Jin, and B. Chen, "A finite element model for static and dynamic analysis of thin-walled beams with asymmetric cross-sections," Computers and Structures, vol. 61, no. 5, pp. 897-908, 1996.

[12] L. Librescu and S. Osheop, Thin-Walled Composite Beams, Theory and Application, Springer, New York, NY, USA, 2006

[13] IACS CSR, International Association of Classification Societies Common structural rules, 2006.

[14] C. S. Smith, Design of Marine Structures in Composite Materials, Elsevier Applied Science, Elsevier Science, New York, NY, USA, 1990.

[15] M. Trautz and R. Herkrath, "The application of folded plate principles on spatial structures with regular, irregular and free-form geometries," in Proceedings of the IASS Symposium, pp. 203-252, Valencia, Spain, 2009.

[16] S. D. Musat and B. I. Epureanu, "Study of warping torsion of thin-walled beams with open crosssection using macro-elements," International Journal for Numerical Methods in Engineering, vol. 44, no. 6, pp. 853-868, 1999.

[17] S. D. Musat, I. Chirica, and V. Giuglea, "Warping torsion analysis of thin-walled closed section beams using macro-elements," in Proceedings of the 3th International Conference on Boundary and Finite Element, vol. 1, pp. 149-158, Constanta, Romania, 1995.

[18] S. D. Musat and B. I. Epureanu, "Study of warping torsion of thin-walled beams with closed crosssection using macro-elements," Communications in Numerical Methods in Engineering, vol. 12, no. 12, pp. 873-884, 1996.

[19] I. Chirica, S. D. Musat, R. Chirica, and E. F. Beznea, “Torsional behaviour of the ship hull composite model," Computational Materials Science, vol. 50, no. 4, pp. 1381-1386, 2011.

[20] S. K. Ider and F. M. L. Amirouche, "Coordinate reduction in the dynamics of constrained multibody systems-a new approach," Journal of Applied Mechanics, vol. 55, no. 4, pp. 899-904, 1988. 